\title{
GRADO DE CONOCIMIENTO Y APLICABILIDAD DE LA FORMACIÓN CONTINUA EN EL TEJIDO \\ EMPRESARIAL GETAFENSE
}

\section{Segundo Valmorisco Pizarro José Luis Herranz Guillén*}

«Los mercados de trabajo locales son los más dinámicos en la creación de empleo. La importancia que tiene eliminar las barreras a la formación para favorecer el resto de las medidas de empleo en estos ámbitos sugieren que los poderes públicos y la sociedad civil tienen que hacer esfuerzos para desarrollar estructuras de formación externas a las empresas, sobre todo para las PYMES» (Sáez,1997)

Los orígenes de la formación continua en España (principios de la década de los 80) coinciden con la consolidación de la democracia, junto con la profundización de la crisis económica (inflación del $9 \%$ anual y tasa de paro del $21 \%{ }^{1}$, siendo en la actualidad del $11,5 \%$ ), y el momento más bajo de la tasa de ocupación (39\%, siendo en la actualidad para el total nacional del $\left.48 \% \%^{2}\right)$. En este escenario es cuando por primera vez representantes políticos y representantes de los agentes sociales asumen compromisos tripartitos sobre la necesidad de poner al día y coordinar las políticas de formación profesional para responder adecuadamente a los nuevos requerimientos del mercado de trabajo.

A su vez, la libre circulación de los trabajadores y el desarrollo del mercado único europeo (por el ingreso de España en la Comunidad Europea) obligó a un gran número de sectores a realizar fuertes ajustes estructurales, de manera que tanto las empresas como los trabajadores tuvieron que hacer frente a profundas

* Segundo Valmorisco Pizarro, Profesor asociado de la Universidad Carlos III, es miembro de la ACMS. José Luis Herranz Guillén es Profesor asociado de la Universidad Carlos III. 
reestructuraciones organizativas y tecnológicas, en un marco en el que la competitividad de las empresas se configuraba como uno de los motores del crecimiento económico y del mantenimiento y generación del empleo. En este sentido, las medidas favorecedoras de la inversión en capital humano se configuraron como políticas necesarias y eficaces para solventar los graves desequilibrios del mercado laboral ${ }^{3}$. Así lo señalaba la Resolución del Consejo de las Comunidades Europeas de 5 de junio de 1989 sobre Formación Profesional Permanente, que perfiló las siguientes funciones principales de la formación continua.

1)Una función de adaptación permanente a la evolución de las profesiones y del contenido de los puestos de trabajo y, por tanto, de mejora de las competencias y las cualificaciones indispensables para fortalecer la situación competitiva de las empresas y de su personal.

2)Una función de promoción social que permita a muchos trabajadores evitar el estancamiento en su cualificación profesional y mejorar su situación laboral.

3)Una función preventiva para anticipar las posibles consecuencias negativas de la realización del mercado interior, y para superar las dificultades que deben afrontar los sectores y las empresas en curso de reestructuración tecnológica.

Sin negar la importancia de otros momentos históricos relevantes en la creación del sistema español de Formación Continua, como la constitución en 1986 del Consejo General de Formación Profesional, o la publicación en 1990 de la Ley de Ordenación General del Sistema Educativo (LOGSE) (que concibe la formación profesional como un único sistema en el que han de integrarse los diferentes subsistemas gestionados), no es hasta 1992 con la firma de los I Acuerdos Nacionales de Formación Continua (ANFC), cuando se puede hablar de una política específica de formación enfocada a los trabajadores ocupados. Anteriormente a esta fecha, los tres subsistemas de formación profesional en España (reglada, ocupacional y continua) recibían un tratamiento transversal e indiferenciado.

A estos primeros acuerdos (1993-1996) siguieron los segundos (1997-2000), y los terceros que están actualmente en vigor (2001-2004), y que han supuesto una paulatina flexibilización en cuanto al acceso de trabajadores y empresas a las subvenciones de la Fundación Tripartita para la Formación en el Empleo ${ }^{4}$, ente público encargado de gestionar la FC en España, compuesto por representantes empresariales, sindicales y de la administración pública.

La aparición de las nuevas tecnologías, la reestructuración del mundo laboral, los problemas del paro, la internacionalización del comercio, el impacto de las comunicaciones, la mutación de los procesos productivos, la amplitud y complejidad de los nuevos conocimientos, la información disponible, el reto de la competitividad, la terciarización de la sociedad, son factores que repercuten en la dinámica del mercado de trabajo y crean la necesidad de adaptación a los nuevos perfiles profesionales que requiere una sociedad y una economía basadas en el conocimiento ${ }^{5}$. 
La evolución que observamos en la planificación de la formación continua en los ámbitos nacional, regional y local, es el producto de estos cambios que se están produciendo en nuestros sistemas económicos y sociales. La velocidad de estos cambios plantea nuevas exigencias a las cualidades de mano de obra que necesitan nuestras empresas, exigencias a las que hay que prestar una especial atención ${ }^{6}$.

Ante este contexto no es descabellado pensar que en un futuro próximo existirá un porcentaje importante de empleos que, según qué actividades, quedarán obsoletos, de ahí la importancia básica de la formación continua.

Por otro lado, existen algunas investigaciones recientes, como Groot (1994) en los Países Bajos y Groot y Oosterbeek (1995) para el Reino Unido, que han encontrado una correlación relevante entre formación continua y productividad de las empresas. Del mismo modo, a medio y largo plazo también se ha advertido una cierta incidencia en la mejora de las ganancias de los trabajadores. Estas investigaciones han demostrado que en promedio la capacitación aumenta la productividad en un $16 \%$ y los salarios entre un $12 \%$ y $15 \%$ respectivamente. Por todo ello, cuando hablamos de formación continua nos estamos refiriendo a una inversión y no a un gasto.

Sin embargo, España debe esforzarse en mayor medida para responder a las directrices para el empleo y las recomendaciones relativas a la prevención y activación. En este sentido, la Comisión de la Unión Europea ${ }^{7}$ recomienda: «elaborar y aplicar una estrategia coherente en materia de formación continua, que fije objetivos e incluya la educación y formación inicial y continua a fin de aumentar los niveles educativos y la participación de los adultos en las actividades de educación y formación».

\section{PLANTEAMIENTO DEL PROBLEMA DE INVESTIGACIÓN}

Desde hace una década en España, y coincidiendo con la firma de los I Acuerdos Nacionales de Formación Continua (diciembre de 1992), los diferentes agentes sociales (organizaciones empresariales y sindicales, y Administraciones Públicas, concretamente el INEM y el Ministerio de Trabajo) están trabajando para conseguir una formación continua de calidad. Sin embargo y sin negar los importantes esfuerzos y logros conseguidos, existen una serie de aspectos que son susceptibles de mejora y que suponen un replanteamiento de algunos capítulos de la FC en nuestro país, para seguir reduciendo la distancia respecto a otros países de la $\mathrm{UE}^{8}$ que poseen unos modelos más antiguos y consolidados que el nuestro, y para seguir haciendo de la FC un vehículo de modernización de las organizaciones. Estos aspectos susceptibles de mejora son visibles en los resultados obtenidos a partir de las memorias elaboradas por FORCEM $^{9}$ en los últimos años: - Se han formado más hombres que mujeres.

- Se han formado más directivos y técnicos y profesionales científicos que trabajadores no cualificados. 
- Los planes de formación han sido orientados desde la oferta formativa y no desde la demanda de los trabajadores.

- Existen carencias en torno a la evaluación de las diferentes acciones formativas, lo que conlleva el desconocimiento de la cualificación que un trabajador asalariado puede incorporar una vez realizado el curso correspondiente.

- Desconocimiento por parte de muchas empresas de las iniciativas de formación financiadas por FORCEM, especialmente en algunas ramas de actividad (por ejemplo en agricultura y pesca).

- Se han formado más trabajadores procedentes de grandes empresas que de PYMES.

Estas conclusiones reflejan que se ha venido realizando una inversión de recursos con una visión más cuantitativa que cualitativa, que lejos de suavizar diferencias han contribuido a perpetuar situaciones.

Una vez constatada esta realidad, el reto que nos planteamos en esta investigación es profundizar en el análisis de estos y otros desequilibrios (indicadores básicos) en algunas empresas getafenses, o dicho de otro modo, comprobar en qué medida estas afirmaciones pueden hacerse extensibles a nuestro municipio.

Además de la aplicabilidad, se ha estudiado también el grado de conocimiento que poseen las empresas encuestadas en torno al modelo de formación continua.

Con todo ello, la Agencia Local de Empleo y Formación (ALEF) presenta este informe de evaluación en el cual se identifican las fortalezas (buenas prácticas) y debilidades de la formación continua en algunas empresas de Getafe. Asimismo, y como veremos en los apartados siguientes, los resultados han sido comparados, en la medida de lo posible, con los obtenidos en: a) la Comunidad de Madrid, a través de las Memorias de la Comisión Paritaria Territorial; b) el ámbito nacional, a través de la Fundación para la Formación Continua; y c) la Unión Europea, mediante las publicaciones de Eurostat.

Esperamos que los resultados sean de utilidad para las empresas colaboradoras y para otras interesadas, y que permitan conocer cuál es el estado de situación en esta materia, cuestión que hasta la fecha no ha sido abordada en el municipio.

\section{METODOLOGÍA}

Para la realización de este estudio se ha escogido una muestra de 50 empresas (en su mayoría PYMES), que en ningún caso pretende ser significativa del universo de unidades del municipio $\left(4.712\right.$ en el año $2000^{10}$ ), lo cual requeriría una muestra muy superior y un trabajo de campo que excede las pretensiones de este estudio introductorio.

De estas 50 empresas que estuvieron dispuestas a participar en el estudio, tan sólo el 24\% (12 empresas) envió el cuestionario o lo respondió por teléfono. Sin 
embargo, y partiendo de los límites propios de cualquier investigación, esperamos que estos resultados (sin riesgo de ser extrapolados al conjunto de unidades locales), permitan establecer un grado de acercamiento mayor a esta realidad en el tejido empresarial de Getafe.

Las empresas se han dividido en dos grandes bloques: por un lado aquellas que han realizado actividades de formación continua, y en segundo lugar aquellas que no lo han hecho nunca. Asimismo, se ha pretendido que queden representadas el mayor número de actividades económicas. El momento de realización de la encuesta fue en los meses de septiembre y octubre de 2002.

Las técnicas utilizadas han sido tanto cuantitativas como cualitativas. Así, se presentó y entregó un cuestionario a las empresas seleccionadas, que contenía preguntas cerradas (que fueron codificadas para su posterior tratamiento estadístico) y abiertas, en las que el encuestador utilizó grabadora. La información recogida a través de esta última técnica fue transcrita y expuesta a análisis semántico.

Asimismo, se han realizado entrevistas en profundidad a diferentes expertos en la materia, que es necesario citar dada su autoridad en los aspectos tratados y su desinteresada participación:

Benítez, María. Agencia Local de Empleo y Formación (ALEF).

Fernández, Eugenio y Lancho, Jaime. Comisiones Obreras (CC.OO).

Jiménez, Pablo. Agrupación Comarcal de Empresarios del Metal (ACEM).

Puerta, Dolores. Unión General de Trabajadores (UGT).

Verdú, Almudena. Agrupación de Comerciantes y Empresarios de Getafe (ACOEG).

Aparte de las técnicas mencionadas, el encuestador portó unas herramientas de información para aquellas empresas que anteriormente no habían realizado formación continua al desconocer las subvenciones públicas que se conceden con cargo a este concepto: direcciones de internet sobre las convocatorias de FORCEM, técnicas para la posterior evaluación en cada empresa, bibliografía básica para conocer el estado de la cuestión en España y en la Comunidad de Madrid, y teléfonos de contacto para la obtención de información por parte de aquellas empresas interesadas en desarrollar actividades de FC.

\section{CARACTERÍSTICAS DEL TEJIDO EMPRESARIAL ENCUESTADO}

En este apartado se recogen datos relativos al tipo de unidad jurídica, actividad económica principal de la empresa encuestada, año de inicio de la actividad en Getafe, número y ubicación geográfica de los establecimientos de la empresa, titularidad, facturación media en los últimos tres años, sistemas de comercialización, número medio de empleados y equipamientos y servicios. 
Las empresas que han participado en el estudio han sido las siguientes:

\begin{tabular}{|c|c|c|}
\hline \multicolumn{2}{|c|}{ EMPRESA } & $\begin{array}{c}\text { ACTIVIDAD ECONÓMICA } \\
\text { PRINCIPAL }\end{array}$ \\
\hline 1 & BBVA & Intermediación financiera \\
\hline 2 & $\begin{array}{c}\text { BIMBO MARTINEZ } \\
\text { COMERCIAL, S.L }\end{array}$ & Industria manufacturera \\
\hline 3 & BRENNTAG QUÍMICAS, S.A & Comercio \\
\hline 4 & $\begin{array}{c}\text { CENTRO DE SALUD "JUAN DE } \\
\text { LA CIERVA" }\end{array}$ & Actividades sanitarias \\
\hline 5 & COJINETES DE FRICCIÓN, S.A & Comercio \\
\hline 6 & CONSTRUCCIONES ARENAL, & Construcción \\
\hline 7 & GESPAL LA PALOMA, S.L & Otras actividades sociales \\
\hline 8 & GRAN CENTRO GETAFE, S.L & Comercio \\
\hline 9 & GUIPUZCOANA & Transporte \\
\hline 10 & EUROEXPRESS, S.A & Educación \\
\hline 11 & KRUSTAGROPUP, S.A & Industria manufacturera \\
\hline 12 & HOTEL LOS OLIVOS & Hostelería \\
\hline
\end{tabular}

La mitad de las empresas encuestadas presentan la personalidad jurídica de sociedad anónima. Si comparamos este dato con el total de unidades locales del municipio, podremos observar que está sobreestimado, ya que según el Directorio de Unidades de Actividad Económica de 2000, elaborado por el Instituto de Estadística de la Comunidad de Madrid, este porcentaje asciende en el municipio de Getafe al 13,1\%. Sin embargo, las sociedades anónimas son las que emplean a un mayor número de personas en Getafe (59,4\% del total de la ocupación), seguidas de las sociedades limitadas, que mantienen el $18,7 \%$ del empleo.

La personalidad jurídica más numerosa que presentan las unidades locales getafenses es, con un 53\% del total de establecimientos, la de "personas físicas" o autónomos. Para responder al interrogante ¿cuál es el compromiso de los trabajadores autónomos con la formación de los trabajadores que emplean?, debemos dirigirnos al II Acuerdo Nacional de Formación Continua, pues en la disposición adicional segunda de dicho Acuerdo, firmado en diciembre de 1997, se introdujo la novedad del acceso de los trabajadores afiliados al régimen especial de autónomos a las subvenciones para el fomento de la formación continua. Sin embargo, la realidad 
nos muestra un índice de participación por parte de este colectivo francamente bajo $^{11}$, en algunos casos debido a su desinterés hacia la formación de sus trabajadores $\mathrm{y}$ en otros debido a su desconocimiento de las subvenciones disponibles ${ }^{12}$.

En cuanto al tamaño, tan sólo hay una gran empresa (Guipuzcoana Euroexpress, S.A), el resto son PYMES ${ }^{13}$. Tampoco hay ninguna microempresa (menos de 10 trabajadores). No es necesario ser gran empresa para presentar un plan de formación a la Fundación Tripartita para la Formación en el Empleo. En el II Acuerdo Nacional de Formación Continua (1997-2000) se incorporó la novedad de reducir el número de trabajadores necesarios que debe tener una empresa para presentar un plan (de 200 a 100 trabajadores), con lo que se facilitó el acceso a las PYMES. No obstante, las pequeñas empresas y las microempresas pueden solicitar planes agrupados. Se trata de planes sectoriales en los que varias empresas de un mismo sector económico, dadas su necesidades y características comunes, se agrupan para solicitar un plan de formación.

La realidad muestra que la tasa de participación de las empresas de 1 a 5 trabajadores en los planes de formación subvencionados con fondos públicos es del 5,4\%, y de 6 a 49 trabajadores es del $22 \%$. A partir de 50 trabajadores, es decir mediana y gran empresa para lo que es la realidad productiva española, dicha tasa nunca es inferior al $40 \%$, llegando al $75,5 \%$ en el caso de las empresas con un estrato de empleo entre 1000 y 4999 trabajadores $^{14}$.

Más del $80 \%$ de las empresas encuestadas presentan titularidad privada. Las organizaciones públicas entrevistadas pertenecen a los sectores de sanidad y educación. En cada acuerdo plurianual (nos encontramos dentro del periodo de vigencia del III ANFC), se firma uno específico para los trabajadores de la administración pública, con lo cual el acceso, en principio, es igualitario en ambos casos, tanto para el sector público como para el privado.

La mayoría de las empresas encuestadas comenzaron su actividad en el municipio a partir del año 1995 (42,7\%), característica que se corresponde, aunque en menor porcentaje, con el total de unidades locales instaladas en Getafe $(35,6 \%)$. Esta considerable cifra de empresas de reciente creación va emparejada a una tasa de supervivencia más bien baja de las empresas después del tercer año, y este problema tiene su explicación entre otras razones, debido a la escasa profesionalización de muchos de quienes ostentan simultáneamente la propiedad y el control de las PYMES en España, cuestión que también afecta de lleno al modelo de formación continua.

Otro grupo importante de empresas de Getafe son las fundadas entre 19801989, que constituyen el 27,1\% del total (si bien en la muestra representan tan sólo el 16,7\%). Muchas de estas deben hacer frente al reciclaje de sus modos de producción, organización y cualificaciones, para adaptarse a la nueva sociedad intensiva en conocimiento y tecnología ${ }^{15}$, pues corren el riesgo de quedar obsoletas al no ponerse al nivel de sus competidoras. 
Debido a las características propias del tejido empresarial encuestado, el $30 \%$ ha facturado en los últimos tres años más de 30 millones de $€$ (5.000 millones de pesetas) de media. Ante esta realidad es pertinente preguntarse ¿cuánto dinero se invierte en la formación de los trabajadores? Es un secreto a voces que muchas empresas que disponen de recursos suficientes prefieren solicitar subvenciones a la Fundación Tripartita, con lo que disminuyen las posibilidades de acceso de las PYMES. Sirva como ejemplo para ilustrar esta idea, la conclusión obtenida a partir de la última memoria de actividades publicada por FORCEM en el año 2000, en la que se observa que el porcentaje de planes de formación solicitados y aprobados para las PYMES es sensiblemente inferior (en torno al 40\%) que en el caso de las grandes empresas (en torno al 60\%).

Asimismo, como se desprende de un estudio realizado por la patronal $\mathrm{CEOE}^{16}$ (encuesta realizada a 600 responsables de formación de empresas españolas de 10 o más trabajadores), el porcentaje de masa bruta salarial dedicado por las empresas españolas a la formación continua es aún muy bajo $(1,9 \%)$ si lo comparamos con otros países europeos como Francia (3,3\% según un estudio realizado por Centre Inffo en 1998). En este sentido se hace necesaria una mayor inversión por parte de las empresas y del Estado, pero no se puede exigir el mismo esfuerzo financiero y administrativo a las PYMES que a las grandes empresas, pues resulta infranqueable para muchas de ellas.

Según una encuesta realizada entre 7.586 empresas por la Asociación para la Formación y el Desarrollo de la Empresa (AFYDE), la formación ocupa el sexto lugar entre las prioridades de las empresas españolas. En esta misma línea, la encuesta de Requerimientos de Empleo y Formación Profesional en las Empresas, realizada por el Ministerio de Trabajo y Seguridad Social en 1993, señalaba que únicamente el $28,1 \%$ de las empresas españolas realiza actividades de formación. Sin embargo, el 45,1\% de ellas declara que sus trabajadores necesitan mayor cualificación "para mejorar su rendimiento en sus puestos de trabajo". La misma fuente muestra una clara influencia del tamaño de la empresa en la programación de la formación continua: el 93,4\% de las empresas de más de 250 trabajadores declara haber realizado alguna acción formativa, frente al $30,4 \%$ de las de menos de 50 trabajadores.

Del mismo modo, en los países europeos más dinámicos existe una importancia creciente en el capítulo de la formación en la empresa, y esto se traduce en una mayor presencia en los convenios colectivos. La opinión de los expertos sindicales del municipio indica que en Getafe esa presencia es aún muy baja.

Francia, país europeo en el que la formación continua se encuentra más desarrollada, es el paradigma de sistema de formación continua muy imbricado en la negociación colectiva. El origen del modelo francés está datado el 9 de julio de 1970 (veintidós años antes que el modelo español), fecha en que los interlocutores sociales sentaron las bases del sistema actual por medio del Acuerdo Nacional 
Interprofesional firmado entre las organizaciones patronales y sindicales. Después sería instaurado el "sistema de formación continua" por la ley del 16 de julio de 1971; dicha ley estableció las bases del sistema de formación continua francés. Lo que distingue a este sistema es el modo en que se combinan los convenios colectivos, los sistemas de paridad y la participación de los interlocutores sociales. El Estado desempeña un papel esencial en la gestión de la formación, arbitrando en las relaciones entre trabajadores y empresa. Asimismo, la ley reconoce formalmente el derecho de los trabajadores a la formación continua.

Los indicadores anteriormente señalados (inversión en formación, interés y necesidad de formar, y presencia en los convenios colectivos), deben hacernos reflexionar sobre la realidad del tejido empresarial getafense y su insuficiente apuesta por la formación continua, sobre todo en el caso de las PYMES.

\section{ESTRUCTURA DEL EMPLEO GENERADO}

En este apartado de la encuesta se le preguntó a las empresas sobre datos muy básicos acerca de los empleados/as: a) sexo, b) grupos de edad, c) tipos de contratos, así como d) empleados según ocupación, e) nivel de estudios, f) áreas funcionales en las que los trabajadores desempeñaban su trabajo. El principal handicap encontrado en este bloque de preguntas ha sido el bajo nivel de respuesta, en especial las tres últimas.

Hecha esta aclaración, las respuestas obtenidas se han distribuido de la siguiente forma: - Por sexo, el 34,7\% de los trabajadores pertenecientes a las empresas encuestadas son mujeres, dato muy similar a la media de la tasa de ocupación femenina que arroja la Encuesta de Población Activa para los municipios de la zona sur de la Comunidad de Madrid (35,1\%). Este porcentaje varía entre el 70\% de media en las empresas encuestadas con titularidad pública (Sanidad y Educación) y el 25,8\% de las empresas con titularidad privada. Tan solo dos empresas privadas superan la media porcentual de empleo femenino en sus plantillas (Hostelería y Otras actividades sociales). Por debajo de la media se encuentran la mayoría de las empresas, destacando alguna de construcción en la que no trabaja ninguna mujer. - Por grupos de edad destaca el colectivo de "entre 25 y 34 años", seguido del "35 a 44 años". Sólo la empresa industrial tiene el mayor porcentaje de plantilla que supera los 45 años. Del mismo modo, las empresas con mayor índice de temporalidad son las que presentan un mayor porcentaje de menores de 25 años en sus plantillas.

- En cuanto al tipo de contrato más utilizado por las empresas encuestadas que han colaborado en el estudio, observamos mayor estabilidad en el empleo (contratos indefinidos) en las empresas pertenecientes al sector industrial, y menor en las empresas de servicios y construcción, en las que algunos casos los contratos temporales ocupan el $100 \%$ del empleo. 
Con estos resultados (baja ocupación femenina y alto índice de temporalidad) surge el interrogante: ¿Cómo se puede intentar llevar acabo una formación cualificante dentro de un modelo de mercado laboral descualificante? Para que la formación continua llegue a todos (sobre todo a las capas más desfavorecidas de la población ocupada) habrá que empezar por cambiar las condiciones laborales imperantes en los mercados de trabajo españoles ${ }^{17}$.

\section{CONOCIMIENTOY YPLICABILIDAD DE LA FORMACIÓN CONTINUA}

Más del 90\% de las empresas encuestadas declaran haber realizado algún tipo de actividad formativa para sus trabajadores desde 1993, otra cuestión es la regularidad. En este sentido y tomando datos de 1999 pertenecientes a la encuesta de formación profesional continua, tan solo el 36\% de las empresas españolas proporciona formación de forma regular a sus empleados ${ }^{18}$, situándose España en este concepto en la penúltima posición europea, sólo por delante de Portugal.Por otro lado, es necesario destacar que algunas empresas declaran que sus trabajadores deben venir ya formados a su puesto de trabajo, ya que desarrollan tareas muy puntuales, y que en su campo no existen avances significativos como para tener que reciclar su capacitación.

En la mayoría de los casos es la propia empresa quien financia la formación con recursos propios y quien imparte dicha formación, en segundo lugar destacan las modalidades mixtas, en las que se combinan la propia empresa, un centro de formación especializado y organizaciones empresariales y/o sindicales.

Cuando hablamos de finalización de la formación continua, el porcentaje aumenta en la modalidad mixta: empresa-organizaciones empresariales y/o sindicales. Al tratarse de una cuestión de inversión de capital, y visto en el primer apartado la facturación media de este conjunto de empresas, el porcentaje mayor es el correspondiente a "la propia empresa", sin embargo, y como ya indicamos anteriormente, muchas empresas con facturación suficiente como para autofinanciar la formación de sus trabajadores utilizan las subvenciones públicas, con lo que limitan el acceso a las que no tienen capacidad de financiársela.

Los cursos para la formación continua de los trabajadores se suelen impartir, en la mayoría de los casos, en el propio centro de trabajo, coincidiendo de este modo con la organización que financia la acción formativa, que es la empresa.

Más del $80 \%$ del tejido empresarial encuestado declara haber realizado un diagnóstico de las necesidades de formación de sus trabajadores, e incluso tener en cuenta estas necesidades para incorporarlas a los planes de formación de la empresa. Diferentes expertos en formación (Felipe Sáez y Cristóbal Torres) ${ }^{19}$ señalan, por el contrario, que se llevan a cabo bastantes más planes de formación de oferta que de demanda, debido a las trabas burocráticas, con lo que es más fácil para la empresa aceptar la oferta formativa que le viene dada que intentar apostar por su propia 
estrategia. En éste sentido, es necesario cuestionarse si la dirección de la empresa está dispuesta a escuchar la opinión de los trabajadores, ya que en muchos casos la formación forma parte de una iniciativa empresarial en la que no suelen participar éstos, a quienes se les aconseja capacitarse para el desempeño de tareas que pueden ayudar a mantener su estabilidad laboral. Del mismo modo, resulta difícil creer que haya PYMES que lleven a cabo detección de necesidades formativas, ya que muchas de ellas no tienen un departamento especializado en gestión de la formación. En este sentido, sería más fácil para ellas recurrir al asociacionismo o habilitar espacios para el diálogo y la investigación de estas cuestiones.

En el extremo opuesto, las empresas deben contar con el riesgo de que haya trabajadores que participen en los programas de formación y abandonen tempranamente la empresa, llevándose consigo ese capital formativo. Asimismo, será difícil para las empresas ajustar su estrategia formativa anticipando los contenidos y las exigencias futuras de los puestos de trabajo, ya que toda incertidumbre equivale a riesgo en mayor o menor grado.

En cualquier caso, señalaremos que la detección de las necesidades de formación es el primer paso para elaborar un plan formativo, y que es muy importante contar con la opinión de los trabajadores, así como conciliar ésta con los propósitos de la dirección para elaborar una estrategia que consiga anticiparse a los cambios futuros.

En efecto, no se trata de que en las PYMES getafenses tenga que haber necesariamente personal dedicado en exclusiva a la formación continua, ya que se trata de algo muy costoso, pero sí que esta sea un área que alguien impulse en la empresa, que forme parte, por ejemplo, de las funciones que desarrolla el directivo responsable de los recursos humanos, ya que menos de la mitad de las empresas encuestadas admiten tener personal que trabaje las cuestiones de formación continua.

En este sentido, es necesario citar el papel integrador que están desarrollando en Getafe algunas organizaciones empresariales como la Agrupación Comarcal de Empresarios del Metal (ACEM) y la Agrupación de Empresarios y Comerciantes de Getafe (ACOEG), al detectar las necesidades de formación de las empresas a quienes representan, industrias maduras y pequeño comercio respectivamente.

La tarea que debe ser encomendada a estos profesionales es la de elaborar itinerarios personalizados de formación (teniendo en cuenta la formación reglada y ocupacional de cada trabajador), y orientar la formación continua de manera que concilie su cualificación con la estrategia de la empresa. La consecuencia que se deriva de este hecho es que las empresas están aceptando sin más la oferta formativa que les llega sin tener la posibilidad de cuestionar si guarda relación o no con sus intereses. Para ello habría que instrumentar las medidas e incentivos necesarios para que los programas tradicionales de oferta fueran sustituidos por una formación "cortada a medida", que se diseñe para responder a las necesidades específicas de las PYMES, a la deseada versatilidad y a los problemas del empleo en cada sector y actividad. 
La mayoría de las empresas encuestadas admiten que el coste económico es la principal dificultad con la que se encuentran a la hora de realizar acciones formativas orientadas a sus trabajadores. Otros argumentos son: les resulta difícil determinar las necesidades formativas, existe falta de interés de los trabajadores y/ o de la empresa, o existe desconocimiento de la oferta formativa existente (véase gráfico 14).

En otro orden de cosas, es necesario señalar que existe una distribución desigual de las acciones de formación continua en las diferentes actividades económicas. Así, en el sector de la automoción y de logística del transporte, en los que se ha producido una renovación tecnológica considerable, la formación ocupa un lugar prioritario. Por el contrario, en la construcción y en la hostelería el porcentaje de participantes es bastante menor, al tratarse de sectores más intensivos en trabajo.

Siendo más del $80 \%$ las empresas que conocen la existencia de FORCEM, tan solo poco más del $40 \%$ ha solicitado subvenciones a la Fundación. Cada una de las empresas tendrá que analizar cuáles son las causas porque solicitan o no subvenciones, cuál es el punto de partida y qué ventajas económicas y financieras conlleva la formación de los trabajadores, tendrá además que analizar a otras empresas de su sector y situar su posición competitiva en el mercado mediante algún tipo de análisis estratégico.

\section{INFORME DE EVALUACIÓN: MODELO KIRKPATRICK PHILLIPS ${ }^{20}$}

Como se ha señalado, la creciente competencia en el mercado nacional y en los mercados mundiales ha llevado a las empresas españolas, madrileñas y getafenses, a una necesidad constante de puesta al día, así como de mejora de sus productos y sus servicios.

Estas circunstancias, así como otras (mejora de las comunicaciones, disminución de los costes de transporte), han tenido y están teniendo un doble efecto: para aquellas empresas preparadas y con sistemas eficientes suponen una gran oportunidad, al encontrarse con menores barreras para acceder a otros mercados. Por el contrario, para las que no han podido o no han sabido situarse en unos niveles adecuados de competitividad, resultan una inquietante amenaza, al acrecentarse los competidores potenciales.

Sea para aprovechar esa oportunidad de crecimiento y desarrollo de la empresa, o para aminorar las posibles amenazas provenientes de otras empresas competidoras, la mejora continua de los productos, los servicios y los procesos, es el camino fundamental para la expansión y la supervivencia, pues sólo a través de esa mejora continua, que resulte en unos incrementos progresivos de los índices de calidad y/o en unos decrementos consecutivos de los costes de los productos o los servicios, pueden ser capaces de resultar competitivas las empresas españolas en general, y las PYMES madrileñas y getafenses en particular. Un adecuado nivel de 
formación permite un mayor grado de destreza y habilidad tanto en los directivos de las empresas como en sus empleados, sin el cual cualquier inversión es ineficiente. Sin el necesario nivel formativo una empresa no puede evolucionar ni competir en entornos abiertamente dinámicos.

Por todo esto se hace necesario evaluar la formación que realizan las empresas. Esta evaluación debe no quedarse sólo en la mera evaluación diagnóstica o de detección de necesidades, sino que es necesario también perfeccionar las técnicas de evaluación sumativas (al final de los procesos de formación), lo que supondría dar un paso más para no evaluar solamente los conocimientos que adquiere un trabajador, sino también la transferencia de estos al puesto de trabajo, así como evaluar el impacto sobre algunos parámetros físicos de explotación (unidades productivas, elementos vendidos, nuevos clientes conseguidos, reducciones de costes y gastos, etc.)

Dicho esto, nuestro propósito en este apartado es evaluar la formación que han llevado acabo las empresas encuestadas de forma agrupada, independientemente del órgano financiador. Aunque sería conveniente que cada empresa realizase su propia evaluación. Para ello existen publicaciones ${ }^{21}$ que pueden facilitar esta tarea.

Para realizar esta evaluación seguiremos el modelo Kirkpatrick Phillips (que es el más divulgado en la evaluación de la formación):

- El primer bloque a evaluar trata específicamente sobre la opinión que les merece a los responsables de recursos humanos las acciones formativas llevadas acabo en la empresa: grado de consecución de los objetivos planteados, contenidos del curso, profesorado y actividades y documentación del curso.

- El segundo bloque hace referencia al grado de conocimiento adquirido por los trabajadores y a la transferencia de esos conocimientos al puesto de trabajo.

- El tercer bloque evalúa el impacto de la formación sobre algunos parámetros físicos de explotación: unidades productivas, elementos vendidos, facturación lograda, nuevos clientes conseguidos y reducciones de costes y gastos debido a la práctica de acciones de formación continua.

\section{BLOQUE PRIMERO}

Siendo la escala 1 el ítem "valoración negativa"y 5 el ítem "valoración positiva", a excepción del porcentaje de valores perdidos, la mayoría de las empresas valoran positivamente la formación recibida por sus trabajadores en cuando a la consecución de los objetivos planteados previamente.

De igual forma se valoran positivamente los contenidos del curso y al profesorado que ha impartido las acciones formativas.

Sin embargo las actividades realizadas y la documentación entregada no recibe una valoración tan positiva. En este sentido las empresas encuestadas argumentan que sería necesario un mayor esfuerzo por parte de quienes imparten la formación en relación a estos dos conceptos. 


\section{BLOQUE SEGUNDO}

En general, existe una valoración positiva de los conocimientos que han adquirido los trabajadores con la formación. Sin embargo, las categorías de respuesta varían más cuando se les ha preguntado sobre la transferencia de estos conocimientos al trabajo cotidiano, lo que lleva a plantearse la idea de la necesaria formación en el puesto de trabajo, incluso de la formación de carácter informal. Sirva como ejemplo el caso de una cadena de montaje para la fabricación de vehículos, en la que el trabajador estará mejor capacitado para el desempeño de tareas si recibe la formación en la misma cadena de montaje en lugar de una clase teórica o a distancia fuera de su lugar de trabajo.

Del mismo modo, se hace necesario integrar la formación aprendida dentro de la cultura y los valores de la empresa (socializar la formación). Esta es una tarea que deben favorecer trabajadores y directivos mediante la mayor implicación de ambos grupos en la planificación estratégica de la formación.

\section{BLOQUE TERCERO}

El siguiente nivel de impacto a evaluar, una vez verificado si los empleados formados ponen en práctica las capacidades aprendidas en sus puestos de trabajo (transferencia), es cuantificar la incidencia que han tenido esas nuevas actitudes y conocimientos en una serie de parámetros o indicadores. El objetivo de esto es verificar en qué medida la formación ha tenido efecto sobre resultados palpables, concretos y visibles. De esta manera, a través de una serie de indicadores podemos observar, de forma cuantificada, la incidencia de la formación sobre estos aspectos, así como su evolución a lo largo del tiempo. Estamos midiendo ahora no tanto el aprendizaje de los individuos sino los efectos que éste ha tenido en la marcha de le empresa.

Es necesario hacer constar que este conjunto de indicadores ha sido respondido tan solo en el 50\% de los casos, lo que indica una escasa familiaridad del tejido empresarial con este tipo de evaluaciones.

En general la valoración realizada por las personas encuestadas es positiva, lo cual indica que existe un alto grado de correlación entre formación y productividad de las empresas.

Si los datos recogidos hasta aquí son positivos, podemos hablar del éxito de la acción formativa, especialmente si los resultados superan a los objetivos prefijados. En caso de que no sea así, debemos proceder a analizar qué causas pueden haber determinado que el resultado no haya sido el esperado. Este desfase entre objetivos y logros puede deberse a la propia formación (en cuyo caso los niveles evaluados previamente deberían haber puesto en evidencia esta circunstancia), o bien a otros aspectos externos que hayan incidido negativamente en la actividad empresarial. Una correcta evaluación de contexto clarificaría mucho esta cuestión. 


\section{CONCLUSIONES}

Teniendo en cuenta los cambios acontecidos en el municipio de Getafe en los últimos años ${ }^{22}$ :

- Aumento del nivel de estudios de la población.

- Subida de las tasas de actividad, especialmente de la población femenina.

- Aparición de importantes cambios demográficos, en los que destaca un progresivo envejecimiento de la población.

- Cambios en los procesos de producción y en la organización del trabajo, que pueden posibilitar el aumento de la formación en el trabajo.

- Prolongación de los itinerarios de "formación a lo largo de toda la vida".

Parece claro que la formación continua va a jugar un papel determinante en el futuro del empleo generado por las empresas de Getafe, sin embargo, y como paso previo, habría que tratar de resolver una serie de aspectos que son susceptibles de mejora, y que suponen un replanteamiento de algunos capítulos de la FC en nuestro país, lo que beneficiaría a la región y a nuestro municipio, y ayudaría a afrontar el futuro de nuestras empresas con mayores garantías.

Del estudio que hemos realizado, así como de opiniones de expertos consultados, podemos concluir que son aspectos susceptibles de mejora los siguientes: 1. Debería producirse un aumento de la inversión en formación continua: el porcentaje de inversión en formación sobre la masa bruta salarial de las empresas es inferior a la de otros países de la Unión Europea, como Holanda, Alemania o Francia. Debe haber una mayor inversión por parte tanto de las empresas como de las Administraciones Públicas, pero no se puede exigir el mismo esfuerzo a las PYMES que a las grandes empresas. Los rediseños futuros del modelo español de FC deberían contemplar esta realidad.

2. En los países europeos más dinámicos se da una importancia creciente en el capítulo de la formación en la empresa y esto se traduce en una mayor presencia en la negociación colectiva. En Getafe, la presencia de cuestiones relacionadas con la FC en la negociación colectiva es todavía muy escasa.

3. Evaluación diagnóstica: detección de necesidades de formación, que ya se está desarrollando dentro del modelo a demanda. Sin embargo, se deben invertir más recursos destinados a la investigación de la evolución de las cualificaciones profesionales. Ello posibilitaría anticipar los cambios y conseguir una formación más acertada, eficaz y eficiente.

4. También es necesario perfeccionar las técnicas de evaluación sumativas (al final de los procesos de formación), lo que supondría dar un paso adelante, al no evaluar solamente los conocimientos que adquiere un trabajador, sino también la transferencia de éstos al puesto de trabajo (unidades productivas, elementos vendidos, nuevos clientes conseguidos, reducciones de costes, mejora de calidad, recursos humanos utilizados, etc). 
5. Es muy importante y urgente potenciar la formación continua en los mercados de trabajo locales, que demuestren ser los más dinámicos desde el punto de vista de la creación de empleo. En este sentido, habrá que realizar ulteriores análisis en profundidad con el objetivo de identificar las ocupaciones en expansión.

6. Se impone armonizar y ensamblar todos los subsistemas (formación reglada, ocupacional y continua) para que respondan a itinerarios personalizados de inserción laboral.

7. No menospreciar la formación continua de tipo informal: se pueden habilitar espacios dentro de la empresa para su desarrollo. Esta es una opción con futuro que ya se está realizando con éxito en otros países europeos, como es el caso de Noruega.

8. Programación flexible: adaptada a las nuevas formas organizativas de la empresa. Es necesario romper con los programas tradicionales "de oferta", y potenciar una formación "cortada a medida", que sea diseñada para corresponder a las necesidades, a la deseada versatilidad y a los problemas idiosincrásicos del empleo.

9. Se debe avanzar en la integración de la formación aprendida con la cultura y el discurso empresarial ("socializar la formación"). Esta es una tarea que deben propiciar trabajadores y empresarios, y vincularla a la filosofía de la calidad total.

10. Todavía existen numerosas empresas que desconocen FORCEM. Para muchas de ellas (sobre todo PYMES) su inversión en innovación y en I+D pasa sólo por la FC subvencionada por la Fundación Tripartita para la Formación en el Empleo, al no disponer de recursos financieros para invertir en procesos de innovación formativa. Igualmente hay que incidir en que autónomos y microempresas aún no conocen FORCEM ${ }^{23}$.

11. Se debe potenciar la formación dirigida a los directivos de las PYMES getafenses, y también para el resto de los trabajadores de las diferentes áreas funcionales, desde técnicos hasta trabajadores no cualificados, colectivo este último, que se enfrenta a importantes discriminaciones en su derecho a formarse dentro de su empresa. Cada agente debe tener una "visión aérea" de su función en la empresa y de los objetivos perseguidos con las diferentes acciones formativas en las que participe. Esto conlleva la necesidad de cambios de mentalidad y nuevas formas de organización del trabajo que han de ser contemplados y articulados en la negociación colectiva.

En resumen, mejorar el acceso a la formación continua de los trabajadores pertenecientes a empresas getafenses es una tarea de todos los agentes que intervienen y participan en el correcto desarrollo del subsistema:

-De FORCEM, accediendo a los mercados de trabajo locales y facilitando el acceso real a PYMES, microempresas y autónomos.

- De las propias empresas, poco concienciadas aún de los beneficios que les pueden reportar la formación continua.

- De los trabajadores, que tienen el derecho de exigir una mayor capacitación, vinculada a una carrera profesional motivadora y cualificadora. 
- De organizaciones empresariales y sindicales, informando y trabajando para conseguir una mayor socialización de la formación continua en Getafe.

La solución, a nuestro juicio, pasa por habilitar espacios para la concertación social. El diálogo entre administración local, sindicatos, empresarios y trabajadores debe ser la base para alcanzar este objetivo en nuestro municipio.

\section{ANÁLISIS ESTRATÉGICO}

\begin{tabular}{|c|c|}
\hline FORTALEZAS & DEBILIDADES \\
\hline $\begin{array}{l}\text { En las empresas encuestadas no se } \\
\text { ha observado discriminación hacia las } \\
\text { mujeres en torno a su acceso a la FC, } \\
\text { tampoco según el nivel formativo o el } \\
\text { área funcional de trabajo. }\end{array}$ & \begin{tabular}{|} 
Los planes de formación de la \\
mayoría de las empresas del municipio \\
están orientados desde la oferta \\
formativa y no tanto desde la \\
demanda de los trabajadores y \\
responsables directivos.
\end{tabular} \\
\hline $\begin{array}{l}\text { En general, los responsables de } \\
\text { recursos humanos de las empresas } \\
\text { getafenses encuestadas están } \\
\text { satisfechos con la formación que } \\
\text { reciben sus trabajadores, y ello se } \\
\text { traduce en la productividad de las } \\
\text { empresas }\end{array}$ & $\begin{array}{c}\text { Escasa práctica de acciones de FC en } \\
\text { trabajadores pertenecientes a empresas } \\
\text { de construcción y hostelería. }\end{array}$ \\
\hline $\begin{array}{l}\text { Según la opinión de los expertos en } \\
\text { formación entrevistados, existe un } \\
\text { aumento creciente de empresas en el } \\
\text { municipio que están comenzando a } \\
\text { considerar la FC y la ponen en práctica. }\end{array}$ & $\begin{array}{l}\text { Sin tener capacidad para especificar el } \\
\text { porcentaje de empresas getafenses que } \\
\text { realizan FC con sus trabajadores, se } \\
\text { estima que este es inferior al } 30 \% \text {, en } \\
\text { cualquier caso una cifra muy baja. }\end{array}$ \\
\hline $\begin{array}{c}\text { Buena disposición de los } \\
\text { representantes empresariales y } \\
\text { sindicales hacía la constitución de } \\
\text { mesas de FC que potencien el dialogo } \\
\text { social. }\end{array}$ & $\begin{array}{c}\text { Baja inversión de las empresas en FC, a } \\
\text { pesar de que la mayoría de las } \\
\text { encuestadas cuentan con capital } \\
\text { suficiente, lo que indica que la FC no es } \\
\text { una prioridad estratégica. }\end{array}$ \\
\hline $\begin{array}{c}\text { Sin pretender entrar en contenidos } \\
\text { formativos (ya que este estudio pretende } \\
\text { identificar "problemas de base"), existen } \\
\text { ocupaciones en Getafe como fresador/a, } \\
\text { tornero/a, etc, que suponen yacimientos } \\
\text { de empleo por los que habría que } \\
\text { apostar con una FC de calidad. }\end{array}$ & $\begin{array}{l}\text { Prácticamente nula presencia de la FC } \\
\text { en los convenios colectivos de las } \\
\text { empresas getafenses. }\end{array}$ \\
\hline
\end{tabular}




\begin{tabular}{|c|c|}
\hline $\begin{array}{c}\text { Se han dado importantes éxitos en el } \\
\text { municipio combinando formación } \\
\text { ocupacional y continua de calidad. } \\
\text { Ejemplo: CINE CITÉ. }\end{array}$ & $\begin{array}{c}\text { Los trámites burocráticos y } \\
\text { administrativos se presentan como un } \\
\text { handicap a la hora de elaborar un plan de } \\
\text { formación. }\end{array}$ \\
\hline $\begin{array}{c}\text { Las empresas líderes de aeronáutica y } \\
\text { componentes de automoción de Getafe } \\
\text { son un ejemplo de buenas prácticas. } \\
\text { Sus resultados deberían trascender más } \\
\text { allá de los límites locales. }\end{array}$ & $\begin{array}{c}\text { Salvo algunas empresas muy } \\
\text { concienciadas, la mayoría no realizan } \\
\text { evaluación (ni inicial ni final), con lo } \\
\text { cual no se conoce el impacto de la } \\
\text { formación en la estrategia empresarial. }\end{array}$ \\
\hline $\begin{array}{c}\text { La potente industria de Getafe y la } \\
\text { estabilidad de su empleo deben ser un } \\
\text { estímulo para que la formación } \\
\text { continua haga este sector aún más } \\
\text { fuerte y competitivo. }\end{array}$ & $\begin{array}{c}\text { Apenas existe socialización de la } \\
\text { formación, en general quien recibe la } \\
\text { formación la aplica sólo individualmente } \\
\text { a sus tareas sin informar al conjunto y } \\
\text { sin espíritu de grupo. }\end{array}$ \\
\hline
\end{tabular}




\section{NOTAS}

${ }^{2}$ Encuesta de Población Activa (4 $4^{\circ}$ trimestre de 1984). Instituto Nacional de Estadística.

${ }^{3}$ Ibid.

${ }^{4}$ Gómez Manzaneque (1997).

${ }^{5}$ Consultar www.forcem. es para obtener más información.

${ }^{6}$ Véase Libro verde Vivir y trabajar en la sociedad del conocimiento: prioridad para las personas. Basado en COM (96) 389 final.

${ }^{7}$ Véase el Estudio sobre la dimensión de la formación continua en España de la Secretaría de Formación y Cultura de Comisiones Obreras (2000).

${ }^{8}$ Véase Recomendaciones del Consejo sobre la aplicación de las políticas de empleo de los estados miembros. COM (99) 445.

${ }^{9}$ Según Eurostat, en España tan sólo el 20\% de los trabajadores ocupados participó en 1993 en acciones de formación continua, siendo la media de la U.E del 28\%. Asimismo, el porcentaje de inversión en formación continua ascendía en España en 1993 al 1\% de la masa bruta salarial, siendo la media de la U.E del 1,6\%.

${ }^{10}$ Memorias de actividades de FORCEM en el nivel estatal (1997-2000) y Memorias de actividades de la Comisión Paritaria Territorial de la Comunidad de Madrid en el nivel regional (1997-1999).

${ }^{11}$ Directorio de unidades de actividad económica. 2000. Instituto de Estadística de la Comunidad de Madrid.

${ }^{12}$ Según la convocatoria de ayudas a la formación continua correspondiente al año 2000, tan sólo el 6,5\% del total de participantes certificados según el tipo de afiliación a la Seguridad Social provenían del Régimen Especial de Autónomos.

${ }^{13}$ Véanse los estudios sectoriales de transporte y madera desarrollados por la Fundación Formación y Empleo Miguel Escalera (FORCEM) de Comisiones Obreras, y "Estudio de las necesidades formativas en el Área del Mantenimiento Preventivo Industrial" desarrollado por el Instituto de Formación y Estudios Sociales (IFES) de la Unión General de Trabajadores.

${ }^{14}$ Se entiende por PYME aquella empresa con menos de 250 trabajadores, con un volumen de negocio anual que no exceda de 40 millones de euros, o balance general anual que no exceda de 27 millones de euros, y que no debe estar participada en más de un $75 \%$ por otra empresa que exceda la condición de PYME (Recomendación europea de 3 de abril de 1996 sobre la definición de pequeñas y medianas empresas. DOCE $n^{\circ}$ L 107 de 30 de abril de 1996).

${ }^{15}$ Véase Memoria FORCEM correspondiente al total de empresas españolas que han realizado formación en el año 2000.

${ }^{16}$ Véase Toffler (1985).

${ }^{17}$ Véase La formación en las empresas españolas, situación, tendencias y expectativas (2002). 
${ }^{18}$ Recientemente (el 10 de noviembre de 2002) la Comisión Europea se ha pronunciado en este sentido, y considera "preocupante" que en España "no se reduzca el nivel de inestabilidad laboral" a pesar de las medidas de fomento del empleo estable acometidas en la última década.

${ }^{19}$ Siendo en el caso de Dinamarca del 96\%, Suecia el 91\% y Holanda el $88 \%$. Sirva además como dato que el tercer país europeo en el que menos formación dispersan las empresas a sus trabajadores es Bélgica con un 70\% (mas del doble que España). Véase Encuesta de Formación Profesional Continua, Nestler y Lailis (1999).

${ }^{20}$ Véase Libro 2000 de la formación. FORCEM (2001).

${ }^{21}$ Véase Kirkpatrick (1999).

${ }^{22}$ Véase Prieto y Carrasco (1999).

${ }^{23}$ Véase Observatorio Ocupacional de la Agencia Local de Empleo y Formación (2001).

${ }^{24}$ FORCEM debe estar más cercana y comprometida con las PYMES, tal y como se extrae del Programa Plurianual de la Empresa y el Espíritu empresarial (20012005) de la Comisión Europea.

\section{BIBLIOGRAFÍA}

AGENCIA PARA LA FORMACIÓN, (2000): Análisis de necesidades de formación en la zona sur de la Comunidad de Madrid, Consejería de Economía y Empleo. Comunidad de Madrid, Madrid.

BURCKEY, R. y CAPLE, J. (1991): La formación. Teoría y práctica, Díaz de Santos, Madrid.

CACHÓN, L. y MONTALVO, M. D. (1999): Educación y formación a las puertas del siglo XXI, Complutense, Madrid.

CEDEFOP (1999): El sistema de formación profesional en España, CEDEFOP. CEDEFOP (2002): Innovations in lifelong learning, Panorama series, 25, CEDEFOP.

CHARRAUD, A. M., MELIVA, A. y PERSONAS, E. (2002): "Organismos de formación continua en Francia. Actividades, objetivos y públicos", en Revista Calificaciones y Empleo, $\mathrm{n}^{\circ} 32$.

COMISIÓN PARITARIA TERRITORIAL DE LA COMUNIDAD DE MADRID. (2001): Memoria de actividades de la aplicación del III ANFC 1999.

EUROSTAT (2002): Continuing vocational training in enterprises in the European Union and Norway, Statistics in Focus.

FERRER DUFOL, J. (2002): Diálogo y concertación social sobre formación en España, CINTERFOR.

FUNDACIÓN COTEC: El sistema español de innovación. Diagnósticos y Recomendaciones. COTEC. 
FUNDACIÓN PARA LA FORMACIÓN CONTÍNUA (2000): Memoria de actividades 1999, FORCEM.

FUNDACIÓN PARA LA FORMACIÓN CONTÍNUA (2001): Memoria de actividades 2000. FORCEM.

FUNDACIÓN PARA LA FORMACIÓN CONTINUA (2002): Políticas y prácticas de la formación continua en el marco europeo, FORCEM.

FUNDACIÓN FORMACIÓN Y EMPLEO MIGUEL ESCALERA (2000): Estudio sobre la dimensión de la formación continua en España, Secretaría de Formación y Cultura de CCOO, Madrid.

FUNDACIÓN TRIPARTITA PARA LA FORMACIÓN EN EL EMPLEO (2002):

Hacia un espacio europeo de aprendizaje permanente, FORCEM.

GÓMEZ MANZANEQUE, B. (1997): "El acuerdo de formación continua (19972000). Invirtiendo en personas, formando el empleo", en Revista del Ministerio de Trabajo y Asuntos Sociales, $\mathrm{n}^{\circ}$ 1, Madrid.

INSTITUTO NACIONAL DE ESTADÍSTICA (2002): Encuesta de Población Activa. Principales resultados (1976-2002), Banco de datos TEMPUS.

KIRKPATRICK. (1999): Evaluación de acciones formativas: los cuatro niveles, EPISE, Barcelona.

MARTÍN ARNÁIZ, J. L. (2001): La formación continua en la estrategia de competitividad de las regiones españolas, XV Reunión Asepelt, A Coruña, 21-22 junio.

MINISTERIO DE EDUCACIÓN, CULTURA Y DEPORTE. (2001): Memorándum sobre el aprendizaje permanente, Documento de trabajo de los servicios de la Comisión, Madrid.

MINISTERIO DE TRABAJO Y ASUNTOS SOCIALES: Encuesta de Formación Profesional Continua. 1999, Madrid.

OBSERVATORIO OCUPACIONAL. (2001): Paro, oferta, demanda y datos de población de Getafe, Agencia Local de Empleo y Formación de Ayuntamiento de Getafe, Madrid.

PARELLADA, M., SÁEZ, F. y TORRES, C. (1999): La formación continua en las empresas españolas y el papel de las universidades, Cívitas, Madrid.

PRIETO, J. M ${ }^{\mathrm{a}}$ y CARRASCO, J.C. (1999): Guía para la evaluación de la formación en las empresas, Complutense, Madrid.

SAÉZ FERNÁNDEZ, F. (1997): "Políticas de formación continua, balance y perspectivas", en Revista del Ministerio de Trabajo y Asuntos Sociales, $\mathrm{n}^{\circ} 1$ Madrid.

TOFFLER, A. (1985): La empresa flexible, Plaza y Janés, Barcelona. 